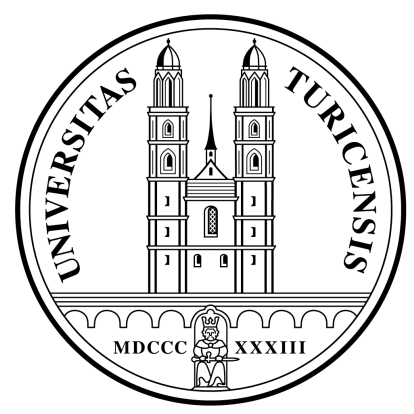

Institute for Empirical Research in Economics

University of Zurich

Working Paper Series

ISSN 1424-0459

Working Paper No. 139

Markets Do Not Select For a Liquidity

Preference as Behavior Towards Risk

Thorsten Hens and Klaus Reiner Schenk-Hoppé

December 2002 


\title{
Markets Do Not Select For a Liquidity Preference as Behavior Towards Risk*
}

\author{
Thorsten Hens ${ }^{\text {a }}$ \\ Klaus Reiner Schenk-Hoppé a,b
}

December 4, 2002

\begin{abstract}
Tobin (1958) has argued that in the face of potential capital losses on bonds it is reasonable to hold cash as a means to transfer wealth over time. It is shown that this assertion cannot be sustained taking into account the evolution of wealth of cash holders versus non cash holders. Cash holders will be driven out of the market in the long run by traders who only use a (risky) long-lived asset to transfer wealth.
\end{abstract}

JEL-Classification: G11, E41, D81.

Keywords: demand for money, portfolio theory, evolutionary finance.

*Financial support by the national centre of competence in research "Financial Valuation and Risk Management" is gratefully acknowledged. The national centers in research are managed by the Swiss National Science Foundation on behalf of the federal authorities.

${ }^{a}$ Institute for Empirical Research in Economics, University of Zurich, Blümlisalpstrasse 10, 8006 Zürich, Switzerland.

${ }^{\mathrm{b}}$ Institute of Economics, University of Copenhagen, Studiestræde 6, DK-1455 Copenhagen K, Denmark.

Email thens@iew.unizh.ch, klaus@econ.ku.dk 


\section{Introduction}

Using two-period mean-variance analysis, Tobin (1958) has argued that in the face of potential capital losses on bonds it is reasonable to hold cash as a means to transfer wealth over time. He concludes on page 66: "If cash is to have any part in the composition of investment balances, it must be because of expectations or fear of loss on other assets." While this assertion is certainly true for two-period models, we argue in this note that it is however hard to sustain in the long run. Analyzing the wealth dynamics resulting from cash and asset holdings we show that in the long run cash holders will be driven out of the market by traders who only use a (risky) long-lived asset to transfer wealth. The main idea of this point is rather simple. The zero return on cash can dominate the return on any other asset with non-negative payoffs only if this asset generates capital losses. That is to say, only if the price of the other asset decreases. The price of an asset having non-negative payoffs can however not decrease below zero. Otherwise the agents would simply withhold the asset from the market and still enjoy the non-negative asset payoffs. Hence capital losses are bounded and eventually the rate of return on the asset will dominate the zero rate of return on money.

Note that on this general level of reasoning, the point of our note is similar to the point made by Hellwig (1993). Hellwig argues that Tobin (1958)'s assertion cannot be sustained in an infinite horizon model with rational expectations. As Hellwig argues, in any point in time money can have a positive value only if some agents believe that the other assets may have sufficiently severe capital losses. Hence in order to have rational expectations in any point in time agents must believe that there is a non-ending sequence of severe capital losses which is inconsistent with positive asset prices.

The point of our note is to show that the general idea of the inconsistency of cash holding in the long run does not need to be based on the assumption of rational expectations. The wealth of all cash holders will eventually become a negligible part of total wealth in the market.

We try to make this point precise in the most simple model. We consider a model in discrete time having a finite state space in every period. The model has two assets. One riskless asset, whose price will be chosen as the numeraire and one risky asset that is risky both in terms of dividends and resale values. Both assets' payoffs are denominated in terms of the numeraire. Hence the payoffs of the assets are in terms of a storable asset and the amount of the numeraire in the model grows over time. Various interpretations of this simple setting are possible. In the case the return on the riskless asset is zero, one may consider the riskless asset as money/cash and one could think of the risky asset as being a console/bond which is exactly the setting 
of Tobin (1958). In the case the return on the riskless asset is positive, one could consider the riskless asset to be a bond while the risky asset may be interpreted as a stock, as it is done in many evolutionary finance models, e.g. Arthur, Holland, LeBaron, Palmer, and Taylor (1997), LeBaron, Arthur, and Palmer (1999), Brock and Hommes (1997), and Lux (1998), among others. Concerning this recent strand of literature our note would suggest to build evolutionary finance models in which cash cannot be used as a store of value. More generally, in our model it turns out that even if the per period payoff of the numeraire is always higher than the dividends paid on the long-lived asset, still - taking into account capital gains - the rate of return on the longlived asset eventually dominates that of the numeraire asset. The reason is that the price of the long-lived asset will grow with the wealth accumulated in the economy. To avoid this feature one must explicitly take into account consumption at a rate at least as high as the growth rate of total payoffs in the market. A simple example of this sort is the famous Lucas (1978) model in which all payoffs are in terms of a single perishable consumption good. Hence in Lucas' model consumption is exactly equal to the inflow of additional units of the numeraire, injected into the model by the assets' payoffs. Our results thus suggest to base evolutionary finance models on Lucas (1978). Models based on this approach are e.g. considered in Blume and Easley $(1992,2001)$, Sandroni (2000), and Evstigneev, Hens, and Schenk-Hoppé (2002).

\section{The Model}

Time is discrete and denoted by $t=0,1,2, \ldots$. There is one long-lived asset and cash. Cash is riskless both in terms of its return $R=1+r \geq 1$ and in terms of its price. It is also used as the numeraire and thus the price of cash is always equal to one. The long-lived asset may be risky both in terms of dividends and resale value. In every period the asset pays off a dividend $D_{t}\left(s^{t}\right) \geq 0$ which is observed at the end of the period. $s^{t}=\left(s_{0}, \ldots, s_{t}\right)$ is the history of states of the world up to period $t, s_{t}$ is the state of the world in period $t$. All payments are in units of cash.

There are $I \geq 2$ agents who can hold cash and the asset to transfer wealth across time. $m_{t}^{i}$ denotes the units of cash hold by investor $i$ at the beginning of period $t$, and $a_{t}^{i}$ denotes the units of the long-lived asset, respectively. As in Tobin (1958) we assume that short selling is not possible. This will in particular rule out negative price bubbles. Alternatively we could have introduced short selling bounded by some arbitrary lower limit.

It is not essential for our reasoning, how the demands $m_{t}^{i}$ and $a_{t}^{i}$ are determined. It could stem from completely rational agents maximizing expected 
utility over infinite horizon, or from boundedly rational agents solving myopic two period maximization problems. It is even allowed to dismiss any rationality interpretation. In this note we consider the evolution of wealth for any sequence of demands $m_{t}^{i}$ and $a_{t}^{i}$ no matter where they come from.

The asset is in fixed supply (normalized to one), while the supply of cash is endogenously given by the cumulated dividends. Thus the market clearing conditions are given by

$$
\sum_{i=1}^{I} a_{t}^{i}=1 \quad \text { and } \quad \sum_{i=1}^{I} m_{t}^{i}=\sum_{\tau=0}^{t} R^{t-\tau} D_{\tau}\left(s^{\tau}\right) \quad \text { for all } t=0,1, \ldots
$$

The wealth of an investor $i$ in period $t$ after dividend payment is determined as

$$
w_{t}^{i}=R m_{t-1}^{i}+\left(D_{t}\left(s_{t}\right)+q_{t}\right) a_{t-1}^{i}
$$

where $q_{t}$ denotes the price of the asset in terms of cash.

The budget constraint of each investor $i$ is

$$
m_{t}^{i}+q_{t} a_{t}^{i}=w_{t}^{i}
$$

Considering the right-hand side of the budget constraint we can already make the intuition of the general argument outlined in the introduction more precise. In the case of cash the net return on the riskless asset is zero, $r=0$. Hence if there are no capital losses, we obtain that whenever the risky asset has some positive payoff, its return dominates that of the riskless asset. Since capital losses are bounded and since the horizon of the model is infinite, eventually cash holding will then be dominated. With a positive net return $r>0$ on the riskless asset, the intuition for our result becomes clear, once the formation of prices has been explained. As we show below, prices increase with market wealth. Hence, the more returns and dividends are paid, the more likely become capital gains on the risky asset. Holdings of agents are described in terms of budget shares. Let $\lambda_{t}^{i}$ denote the fraction of wealth an investor $i$ assigns to the purchase of the asset and let $1-\lambda_{t}^{i}$ denote the fraction of wealth assigned to cash holdings, i.e.

$$
m_{t}^{i}=\left(1-\lambda_{t}^{i}\right) w_{t}^{i} \quad \text { and } \quad a_{t}^{i}=\frac{\lambda_{t}^{i} w_{t}^{i}}{q_{t}}
$$

Rewriting (2) one obtains

$$
w_{t}^{i}=R\left(1-\lambda_{t-1}^{i}\right) w_{t-1}^{i}+\left(D_{t}\left(s_{t}\right)+q_{t}\right) \frac{\lambda_{t-1}^{i} w_{t-1}^{i}}{q_{t-1}}
$$


Equation (4) implies that the market-clearing price $q_{t}$ is given by

$$
q_{t}=\sum_{i} \lambda_{t}^{i} w_{t}^{i}=\lambda_{t} w_{t}
$$

where $\lambda_{t}=\left(\lambda_{t}^{1}, \ldots, \lambda_{t}^{I}\right)$ and $w_{t}^{T}=\left(w_{t}^{1}, \ldots, w_{t}^{I}\right)$. If for some investor $\lambda_{t}^{i} w_{t}^{i}>0$, then $q_{t}>0$. Since wealth increases with returns on the riskless and on the risky asset, prices are more likely to rise when the riskless asset has positive returns. Hence in that case capital losses are even less likely than in the case of money/cash.

Inserting (6) in (5) yields an implicit equation for the wealth of investor $i$ in period $t$ for each given distribution of wealth across investors $w_{t-1}$ in period $t-1$. Define

$$
A_{t-1}^{i}=R\left(1-\lambda_{t-1}^{i}\right) w_{t-1}^{i}+D_{t}\left(s^{t}\right) B_{t-1}^{i}
$$

and

$$
B_{t-1}^{i}=\frac{\lambda_{t-1}^{i} w_{t-1}^{i}}{\lambda_{t-1} w_{t-1}}
$$

The subscript $t-1$ refers to the time-dependence of the wealth distribution $w_{t-1}$. This implicit equation for the evolution of wealth can now be written as

$$
w_{t}=A_{t-1}+B_{t-1} \lambda_{t} w_{t}
$$

with $A_{t-1}^{T}=\left(A_{t-1}^{1}, \ldots, A_{t-1}^{I}\right)$ and $B_{t-1}^{T}=\left(B_{t-1}^{1}, \ldots, B_{t-1}^{I}\right)$. One needs to solve (7) for $w_{t}$ to derive the law of motion for the distribution of wealth across investors.

¿From (7) we obtain

$$
w_{t}=\left(I-B_{t-1} \lambda_{t}\right)^{-1} A_{t-1}
$$

where $I$ is the identity. The inverse of $I-B_{t-1} \lambda_{t}$ is given by $I+(1-$ $\left.\lambda_{t} B_{t-1}\right)^{-1} B_{t-1} \lambda_{t}$ provided $\lambda_{t} B_{t-1} \neq 1$ (Horn and Johnson 1985, Sec. 0.7.4). It is straightforward to check that in our model $\lambda_{t} B_{t-1}<1$, if for some investor $\lambda_{t}^{i}<1$ and $\lambda_{t-1}^{i} w_{t-1}^{i}>0$.

One finally obtains

$$
w_{t}=\left(I+\frac{1}{1-\lambda_{t} B_{t-1}} B_{t-1} \lambda_{t}\right) A_{t-1}
$$

where the $i$ th component of (9) is given by

$$
\begin{aligned}
w_{t}^{i}=( & R\left(1-\lambda_{t-1}^{i}\right)+D_{t}\left(s^{t}\right) \frac{\lambda_{t-1}^{i}}{\lambda_{t-1} w_{t-1}} \\
& \left.+\lambda_{t-1}^{i} \frac{\sum_{j}\left[R\left(1-\lambda_{t-1}^{j}\right)+D_{t}\left(s^{t}\right) \frac{\lambda_{t-1}^{j}}{\lambda_{t-1} w_{t-1}}\right] \lambda_{t}^{j} w_{t-1}^{j}}{\sum_{j}\left(1-\lambda_{t}^{j}\right) \lambda_{t-1}^{j} w_{t-1}^{j}}\right) w_{t-1}^{i}
\end{aligned}
$$


It is clear from the above discussion and from (9) that the evolution of the wealth distribution is well defined if at least one investor $i$ with initial wealth $w_{0}^{i}>0$ adopts an investment rule with $\lambda_{t}^{i} \in(0,1)$ for all $t$.

\section{The Main Result}

Tobin (1958)'s assertion on the rationality of holding cash in the presence of potential capital losses is now addressed in the model introduced above for the particular case of two investors. The first investor only holds the risky asset to transfer wealth across time $\left(\lambda_{t}^{1}=1\right)$ while the second investor holds a mixed portfolio and invests partly in cash $\left(0<\lambda_{t}^{2}<1\right)$. Both investors are endowed with initial wealth $w_{0}^{i}>0$. Under these two assumptions the equation governing the evolution of wealth (9) is well-defined. The model with $I=2$ turns out to be analytically tractable because the inverse matrix in (9) has a simple expression. In particular one can study the long-run distribution of wealth in this case.

After some lengthy but elementary calculations (which can be found in the appendix) one obtains that, for $I=2,(9)$ is equivalent to

$$
\begin{aligned}
w_{t}^{1} & =\frac{D_{t}\left(s^{t}\right)+R\left(1-\lambda_{t-1}^{2}\right) \lambda_{t}^{2} w_{t-1}^{2}}{\left(1-\lambda_{t}^{2}\right) \lambda_{t-1}^{2} w_{t-1}^{2}} w_{t-1}^{1} \\
w_{t}^{2} & =\frac{D_{t}\left(s^{t}\right)+R\left(1-\lambda_{t-1}^{2}\right) w_{t-1}^{2}}{\left(1-\lambda_{t}^{2}\right) w_{t-1}^{2}} w_{t-1}^{2}
\end{aligned}
$$

¿From equations (10) we can analyze the possibility of capital losses on the risky asset. To this end compute its price change, which is, by (6), $q_{t}-q_{t-1}=$ $w_{t}^{1}+\lambda_{t}^{2} w_{t}^{2}-\left(w_{t-1}^{1}+\lambda_{t-1}^{2} w_{t-1}^{2}\right)$. Inserting (10) one obtains

$$
\begin{aligned}
q_{t}-q_{t-1}= & \frac{D_{t}\left(s^{t}\right)+\left(\left[1+\left(1-\lambda_{t-1}^{2}\right) r\right] \lambda_{t}^{2}-\lambda_{t-1}^{2}\right) w_{t-1}^{2}}{\left(1-\lambda_{t}^{2}\right) \lambda_{t-1}^{2} w_{t-1}^{2}} w_{t-1}^{1} \\
& +\frac{\lambda_{t}^{2} D_{t}\left(s^{t}\right)+\left(\left[1+\left(1-\lambda_{t-1}^{2}\right) r\right] \lambda_{t}^{2}-\lambda_{t-1}^{2}\right) w_{t-1}^{2}}{\left(1-\lambda_{t}^{2}\right) w_{t-1}^{2}} w_{t-1}^{2}
\end{aligned}
$$

If dividends on the risky asset were negligible, capital losses would occur if the second agents' budget share for the risky asset $\lambda_{t}^{2}$ decreases by more than the riskfree return $R=1+r$. In particular from this expression we see that capital losses are more likely if the riskfree return $r$ is zero.

Since there is no consumption, the total wealth $W_{t}=w_{t}^{1}+w_{t}^{2}$ of the economy may become arbitrarily large as time tends to infinity. In this case the dividends may become negligible in the long-run. For instance, 
it is apparent from (1) and (3) that the aggregate wealth tends to infinity (almost surely) if dividend payments $\sum_{\tau=0}^{t} D_{\tau}\left(s^{\tau}\right) \rightarrow \infty$ (almost surely): $W_{t} \geq m_{t}^{1}+m_{t}^{2}=\sum_{\tau=0}^{t} R^{t-\tau} D_{\tau}\left(s^{\tau}\right) \geq \sum_{\tau=0}^{t} D_{\tau}\left(s^{\tau}\right)$, since $R \geq 1$. If dividends are uniformly bounded from above, the dividend-wealth ratio, $D_{t}\left(s^{t}\right) / W_{t}$, converges to zero.

The most convenient way to avoid this problem is to make the following assumption:

(A) $D_{t}\left(s^{t}\right)=d\left(s_{t}\right) W_{t-1}$ and $d\left(s_{t}\right) \geq 0$ is an ergodic process such that $d(s)>0$ with positive probability.

That is the dividend grows on average with the same rate as the economy.

Under assumption (A) one obtains from (10) an equation for the evolution of the ratio of the investors' wealth shares $r_{t}^{i}=w_{t}^{i} / W_{t}$ :

$$
\left(\frac{w_{t}^{1}}{w_{t}^{2}}=\right) \frac{r_{t}^{1}}{r_{t}^{2}}=\frac{1}{\lambda_{t-1}^{2}} \frac{d\left(s_{t}\right)+R\left(1-\lambda_{t-1}^{2}\right) \lambda_{t}^{2} r_{t-1}^{2}}{d\left(s_{t}\right)+R\left(1-\lambda_{t-1}^{2}\right) r_{t-1}^{2}} \cdot \frac{r_{t-1}^{1}}{r_{t-1}^{2}}
$$

We make the assumption

(B) There is a $\delta>0$ such that $\delta \leq \lambda_{t}^{2} \leq 1-\delta$ for all $t$.

Under this assumption investor 2 cannot mimic investor 1's investment strategy who only holds the risky asset. In fact it suffices to require that the fraction of wealth allocated to the purchase of the risky asset by investor 2 does not tend to zero or one exponentially fast.

Theorem 1 Under assumptions (A) and (B) the investor holding only the risky asset (while the other investor also holds cash) gathers total wealth almost surely. The investor with a mixed portfolio becomes extinct.

Hence we have shown that while Tobin (1958)'s argument for a liquidity preference as behavior towards risk certainly makes sense in the short run, it is not sustainable in the long run if one takes the wealth dynamics into account. Moreover, our result may be seen as a justification of building evolutionary models analyzing the market selection hypothesis based on the ideas of Lucas (1978). If, as in Lucas (1978), asset payoffs are denominated in terms of a perishable consumption good rather than in terms of some of the assets then these payoffs do not drive out the asset in which payoffs are denominated as a reasonable investment alternative vis a vis the other assets. For example in Evstigneev, Hens, and Schenk-Hoppé (2002) a riskless asset with positive payoff $R$ that is dominated by the the payoff $D$ of some other 
asset can well be sustained as an evolutionary stable outcome of the wealth dynamics.

Proof of Theorem 1 The task in this proof is mainly to derive a lower bound on the asymptotic growth rate of the market share ratio $r_{t}^{1} / r_{t}^{2}$. It will be shown that for any investment strategy $\left(\lambda_{t}^{2}\right)_{t \geq 0}$ the asymptotic growth rate $\lim _{t \rightarrow \infty} 1 / t \ln \left(r_{t}^{1} / r_{t}^{2}\right)>0$. This implies (as is detailed below) $r_{t}^{1} \rightarrow 1$ and $r_{t}^{2}=1-r_{t}^{1} \rightarrow 0$. Thus investor 1 (who invests only in the risky asset) gathers total market wealth in the long run.

Consider the right-hand side of (12). Let us first show that for each fixed $d \geq 0$

$$
\frac{d+R\left(1-\lambda_{t-1}^{2}\right) \lambda_{t}^{2} r_{t-1}^{2}}{d+R\left(1-\lambda_{t-1}^{2}\right) r_{t-1}^{2}} \geq \lambda_{t}^{2}+\alpha
$$

with $\alpha \geq 0$ (and $\alpha>0$ if $d>0$ ) for all $\delta \leq \lambda_{t-1}^{2} \leq 1-\delta$ and $0<r_{t-1}^{2} \leq 1$. (13) is equivalent to

$$
\alpha \leq \frac{\left(1-\lambda_{t}^{2}\right) d}{d+R\left(1-\lambda_{t-1}^{2}\right) r_{t-1}^{2}}
$$

The right-hand side of (14) is decreasing in $r_{t-1}^{2}$ as well as in $\lambda_{t}^{2}$ and increasing in $\lambda_{t-1}^{2}$. Inserting the maximal resp. minimal possible values for these variables a sufficient condition on $\alpha$ is obtained:

$$
\alpha \leq \frac{\delta d}{d+R(1-\delta)}
$$

For each $d \geq 0$, let us define $\alpha(d) \geq 0$ by the right-hand side of (15).

Taking the derivative with respect to $d$ it is straightforward to see that $\alpha(d)$ is increasing in $d$.

Fixing any $\varepsilon>0,(13)$ thus implies that for every $d\left(s_{t}\right) \geq \varepsilon$,

$$
\frac{1}{\lambda_{t-1}^{2}} \frac{d\left(s_{t}\right)+R\left(1-\lambda_{t-1}^{2}\right) \lambda_{t}^{2}}{d\left(s_{t}\right)+R\left(1-\lambda_{t-1}^{2}\right)} \geq \frac{\lambda_{t}^{2}+\bar{\alpha}}{\lambda_{t-1}^{2}}
$$

with $\bar{\alpha}=\alpha(\varepsilon)$. From (13) and the fact that $\alpha(d) \geq 0$ we also find that for all $d\left(s_{t}\right)$

$$
\frac{1}{\lambda_{t-1}^{2}} \frac{d\left(s_{t}\right)+R\left(1-\lambda_{t-1}^{2}\right) \lambda_{t}^{2}}{d\left(s_{t}\right)+R\left(1-\lambda_{t-1}^{2}\right)} \geq \frac{\lambda_{t}^{2}}{\lambda_{t-1}^{2}}
$$

Summarizing these findings, we obtain the following estimate from below on $(12)$ :

$$
\frac{r_{t}^{1}}{r_{t}^{2}} \geq\left(\mathbf{1}_{d_{t} \geq \varepsilon} \frac{\lambda_{t}^{2}+\bar{\alpha}}{\lambda_{t-1}^{2}}+\mathbf{1}_{d_{t}<\varepsilon} \frac{\lambda_{t}^{2}}{\lambda_{t-1}^{2}}\right) \frac{r_{t-1}^{1}}{r_{t-1}^{2}}
$$


where $\mathbf{1}_{d_{t} \geq \varepsilon} \in\{0,1\}$ with $\mathbf{1}_{d_{t} \geq \varepsilon}=1$ if and only if $d\left(s_{t}\right) \geq \varepsilon$. Analogously for $\mathbf{1}_{d_{t}<\varepsilon}$. Taking logarithms, we find

$$
\ln \frac{r_{t}^{1}}{r_{t}^{2}} \geq \sum_{\tau=1}^{t} \ln \left(\frac{\mathbf{1}_{d_{\tau} \geq \varepsilon}\left(\lambda_{\tau}^{2}+\bar{\alpha}\right)+\mathbf{1}_{d_{\tau}<\varepsilon} \lambda_{\tau}^{2}}{\lambda_{\tau-1}^{2}}\right)+\ln \frac{r_{0}^{1}}{r_{0}^{2}}
$$

The sum on the right-hand side can be estimated from below as

$$
\begin{gathered}
\sum_{\tau=1}^{t} \ln \left(\frac{\mathbf{1}_{d_{\tau} \geq \varepsilon}\left(\lambda_{\tau}^{2}+\bar{\alpha}\right)+\mathbf{1}_{d_{\tau}<\varepsilon} \lambda_{\tau}^{2}}{\lambda_{\tau-1}^{2}}\right)=\ln \prod_{\tau=1}^{t}\left(\frac{\mathbf{1}_{d_{\tau} \geq \varepsilon}\left(\lambda_{\tau}^{2}+\bar{\alpha}\right)+\mathbf{1}_{d_{\tau}<\varepsilon} \lambda_{\tau}^{2}}{\lambda_{\tau-1}^{2}}\right) \\
=\ln \left[\frac{\mathbf{1}_{d_{t} \geq \varepsilon}\left(\lambda_{t}^{2}+\bar{\alpha}\right)+\mathbf{1}_{d_{t}<\varepsilon} \lambda_{t}^{2}}{\lambda_{0}^{2}} \cdot \prod_{\tau=1}^{t-1}\left(\mathbf{1}_{d_{\tau} \geq \varepsilon} \frac{1+\bar{\alpha}}{\lambda_{\tau-1}^{2}}+\mathbf{1}_{d_{\tau}<\varepsilon}\right)\right] \\
=\ln \frac{\mathbf{1}_{d_{t} \geq \varepsilon}\left(\lambda_{t}^{2}+\bar{\alpha}\right)+\mathbf{1}_{d_{t}<\varepsilon} \lambda_{t}^{2}}{\lambda_{0}^{2}}+\sum_{\tau=1}^{t-1} \ln \left(\mathbf{1}_{d_{\tau} \geq \varepsilon} \frac{1+\bar{\alpha}}{\lambda_{\tau-1}^{2}}+\mathbf{1}_{d_{\tau}<\varepsilon}\right) \\
\geq \ln \frac{\lambda_{t}^{2}}{\lambda_{0}^{2}}+\sum_{\tau=1}^{t-1} \ln \left(\mathbf{1}_{d_{\tau} \geq \varepsilon} \frac{1+\bar{\alpha}}{1-\delta}+\mathbf{1}_{d_{\tau}<\varepsilon}\right) \geq \ln \frac{\delta}{1-\delta}+C \sum_{\tau=1}^{t-1} \mathbf{1}_{d_{\tau} \geq \varepsilon}
\end{gathered}
$$

where $C=\ln [1+\bar{\alpha} /(1-\delta)]>0$.

The long-run growth rate of $r_{t}^{1} / r_{t}^{2}$ is thus bounded from below by

$$
\lim _{t \rightarrow \infty} \frac{1}{t} \ln \frac{r_{t}^{1}}{r_{t}^{2}} \geq \lim _{t \rightarrow \infty} \frac{1}{t}\left(\ln \frac{\delta}{1-\delta}+C \sum_{\tau=1}^{t-1} \mathbf{1}_{d_{\tau} \geq \varepsilon}+\ln \frac{r_{0}^{1}}{r_{0}^{2}}\right)=C \mathbf{P}\{d(s) \geq \varepsilon\}
$$

where the last equality follows from the ergodic theorem.

Assumption (A) implies $\mathbf{P}\{d(s) \geq \varepsilon\}>0$ for all sufficiently small $\varepsilon>0$. Since $C>0$ for every fixed $\varepsilon>0$, the last equation implies

$$
\lim _{t \rightarrow \infty} \frac{1}{t} \ln \frac{r_{t}^{1}}{r_{t}^{2}}=: \gamma>0
$$

for all small enough $\varepsilon>0$. This means for $t$ large

$$
\frac{r_{t}^{1}}{1-r_{t}^{1}}=\frac{r_{t}^{1}}{r_{t}^{2}} \approx \exp (t \gamma) \rightarrow \infty \text { as } t \rightarrow \infty
$$

which implies $r_{t}^{1} \rightarrow 1$ (and $r_{t}^{2} \rightarrow 0$ ) as $t \rightarrow \infty$ almost surely. Convergence is even exponentially fast. This completes the proof of Theorem 1 . 


\section{References}

Arthur, W. B., J. H. Holland, B. LeBaron, R. G. Palmer, and P. TAYLOR (1997): "Asset Pricing under Endogenous Expectations in an Artificial Stock Market," in The Economy as an Evolving Complex System II, ed. by W. B. Arthur, S. Durlauf, and D. Lane, pp. 15-44. Addison Wesley.

Blume, L., AND D. EAsley (1992): "Evolution and Market Behavior," Journal of Economic Theory, 58, 9-40.

(2001): "If You Are So Smart Why Aren't You Rich? Belief Selection in Complete and Incomplete Markets," Mimeo, Cornell University.

Brock, W. A., and C. H. Hommes (1997): "A Rational Route to Randomness," Econometrica, 65, 1059-1095.

Evstigneev, I., T. Hens, and K. R. Schenk-Hoppé (2002): "Market Selection of Financial Trading Strategies: Global Stability," Mathematical Finance, 12, 329-339.

Hellwig, M. F. (1993): "The Challenge of Monetary Theory," European Economic Review, 37, 215-242.

Horn, R. A., And C. R. Johnson (1985): Matrix Analysis. Cambridge University Press, Cambridge UK.

LeBaron, B., W. B. Arthur, and R. Palmer (1999): "Time Series Properties of an Artificial Stock Market," Journal of Economic Dynamics and Control, 23, 1487-1516.

LuCAs, R. (1978): "Asset Prices in an Exchange Economy," Econometrica, 46, 1429-1445.

Lux, T. (1998): "The Socio-Economic Dynamics of Speculative Markets: Interacting Agents, Chaos, and the Fat Tails of Return Distributions," Journal of Economic Behavior and Organization, 33, 143-165.

SAndroni, A. (2000): "Do Markets Favor Agents Able to Make Accurate Predictions?," Econometrica, 68, 1303-1341.

ToBin, J. (1958): "Liquidity Preference as Behavior Towards Risk," The Review of Economic Studies, 25, 65-86. 


\section{Appendix}

The following elementary calculations show that (9) and (10) are equivalent.

Let $I=2$. Further, let $\lambda_{t}^{1}=1$ and $0<\lambda_{t}^{2}<1$.

For the first investor $i=1$ we have

$$
w_{t}^{1}=\left(\frac{D_{t}\left(s^{t}\right)}{\lambda_{t-1} w_{t-1}}+\frac{\frac{D_{t}\left(s^{t}\right)}{\lambda_{t-1} w_{t-1}} w_{t-1}^{1}+\left[R\left(1-\lambda_{t-1}^{2}\right)+D_{t}\left(s^{t}\right) \frac{\lambda_{t-1}^{2}}{\lambda_{t-1} w_{t-1}}\right] \lambda_{t}^{2} w_{t-1}^{2}}{\left(1-\lambda_{t}^{2}\right) \lambda_{t-1}^{2} w_{t-1}^{2}}\right) w_{t-1}^{1}
$$

A sequence of equivalent expressions is derived by elementary calculations

$$
\begin{aligned}
w_{t}^{1}= & \left(D_{t}\left(s^{t}\right)+\frac{\left.D_{t}\left(s^{t}\right) w_{t-1}^{1}+\left[R\left(1-\lambda_{t-1}^{2}\right) \lambda_{t-1} w_{t-1}+D_{t}\left(s^{t}\right) \lambda_{t-1}^{2}\right] \lambda_{t}^{2} w_{t-1}^{2}\right)}{\left(1-\lambda_{t}^{2}\right) \lambda_{t-1}^{2} w_{t-1}^{2}}\right) \frac{w_{t-1}^{1}}{\lambda_{t-1} w_{t-1}} \\
w_{t}^{1}= & \frac{1}{\left(1-\lambda_{t}^{2}\right) \lambda_{t-1}^{2} w_{t-1}^{2}} \frac{w_{t-1}^{1}}{\lambda_{t-1} w_{t-1}} \times \\
& \left.+D_{t}\left(s^{t}\right) w_{t-1}^{1}+\left[R\left(1-\lambda_{t-1}^{2}\right) \lambda_{t-1} w_{t-1}+D_{t}\left(s^{t}\right) \lambda_{t-1}^{2}\right] \lambda_{t}^{2} w_{t-1}^{2}\right) \\
w_{t}^{1}= & \frac{1}{\left(1-\lambda_{t}^{2}\right) \lambda_{t-1}^{2} w_{t-1}^{2}} \frac{w_{t-1}^{2}}{\lambda_{t-1} w_{t-1}^{2}} \times \\
& \left(D_{t}\left(s^{t}\right) \lambda_{t-1}^{2} w_{t-1}^{2}-D_{t}\left(s^{t}\right) \lambda_{t}^{2} \lambda_{t-1}^{2} w_{t-1}^{2}\right. \\
w_{t}^{1}= & \frac{1}{\left(1-\lambda_{t}^{2}\right) \lambda_{t-1}^{2} w_{t-1}^{2}} \frac{\left.\left.w_{t-1}^{t}\right) w_{t-1}^{1}+R\left(1-\lambda_{t-1}^{2}\right) \lambda_{t-1} w_{t-1} \lambda_{t}^{2} w_{t-1}^{2}+D_{t}\left(s^{t}\right) \lambda_{t-1}^{2} \lambda_{t}^{2} w_{t-1}^{2}\right)}{\left.D_{t}\left(s^{t}\right) \lambda_{t-1} w_{t-1}+R\left(1-\lambda_{t-1}^{2}\right) \lambda_{t-1} w_{t-1} \lambda_{t}^{2} w_{t-1}^{2}\right)}
\end{aligned}
$$

Finally, this is equivalent to

$$
w_{t}^{1}=\frac{D_{t}\left(s^{t}\right)+R\left(1-\lambda_{t-1}^{2}\right) \lambda_{t}^{2} w_{t-1}^{2}}{\left(1-\lambda_{t}^{2}\right) \lambda_{t-1}^{2} w_{t-1}^{2}} w_{t-1}^{1}
$$


Next, consider the second investor $i=2$

$$
\begin{aligned}
& w_{t}^{2}=( R\left(1-\lambda_{t-1}^{2}\right)+\frac{D_{t}\left(s^{t}\right) \lambda_{t-1}^{2}}{\lambda_{t-1} w_{t-1}} \\
&\left.\quad+\lambda_{t-1}^{2} \frac{\frac{D_{t}\left(s^{t}\right)}{\lambda_{t-1} w_{t-1}} w_{t-1}^{1}+\left[R\left(1-\lambda_{t-1}^{2}\right)+D_{t}\left(s^{t}\right) \frac{\lambda_{t-1}^{2}}{\lambda_{t-1} w_{t-1}}\right] \lambda_{t}^{2} w_{t-1}^{2}}{\left(1-\lambda_{t}^{2}\right) \lambda_{t-1}^{2} w_{t-1}^{2}}\right) w_{t-1}^{2}
\end{aligned}
$$

Again we make some elementary transformations

$$
\begin{aligned}
& w_{t}^{2}=\left(R\left(1-\lambda_{t-1}^{2}\right) \lambda_{t-1} w_{t-1}+D_{t}\left(s^{t}\right) \lambda_{t-1}^{2}\right. \\
& \left.+\lambda_{t-1}^{2} \frac{D_{t}\left(s^{t}\right) w_{t-1}^{1}+\left[R\left(1-\lambda_{t-1}^{2}\right) \lambda_{t-1} w_{t-1}+D_{t}\left(s^{t}\right) \lambda_{t-1}^{2}\right] \lambda_{t}^{2} w_{t-1}^{2}}{\left(1-\lambda_{t}^{2}\right) \lambda_{t-1}^{2} w_{t-1}^{2}}\right) \frac{w_{t-1}^{2}}{\lambda_{t-1} w_{t-1}} \\
& w_{t}^{2}=\frac{1}{\left(1-\lambda_{t}^{2}\right) \lambda_{t-1}^{2} w_{t-1}^{2}} \frac{w_{t-1}^{2}}{\lambda_{t-1} w_{t-1}} \times \\
& \left(\left[R\left(1-\lambda_{t-1}^{2}\right) \lambda_{t-1} w_{t-1}+D_{t}\left(s^{t}\right) \lambda_{t-1}^{2}\right]\left(1-\lambda_{t}^{2}\right) \lambda_{t-1}^{2} w_{t-1}^{2}\right. \\
& \left.+\lambda_{t-1}^{2} D_{t}\left(s^{t}\right) w_{t-1}^{1}+\lambda_{t-1}^{2}\left[R\left(1-\lambda_{t-1}^{2}\right) \lambda_{t-1} w_{t-1}+D_{t}\left(s^{t}\right) \lambda_{t-1}^{2}\right] \lambda_{t}^{2} w_{t-1}^{2}\right) \\
& w_{t}^{2}=\frac{1}{\left(1-\lambda_{t}^{2}\right) \lambda_{t-1}^{2} w_{t-1}^{2}} \frac{w_{t-1}^{2}}{\lambda_{t-1} w_{t-1}} \times \\
& \left(R\left(1-\lambda_{t-1}^{2}\right) \lambda_{t-1} w_{t-1}\left(1-\lambda_{t}^{2}\right) \lambda_{t-1}^{2} w_{t-1}^{2}+D_{t}\left(s^{t}\right) \lambda_{t-1}^{2}\left(1-\lambda_{t}^{2}\right) \lambda_{t-1}^{2} w_{t-1}^{2}\right. \\
& \left.+\lambda_{t-1}^{2} D_{t}\left(s^{t}\right) w_{t-1}^{1}+\lambda_{t-1}^{2} R\left(1-\lambda_{t-1}^{2}\right) \lambda_{t-1} w_{t-1} \lambda_{t}^{2} w_{t-1}^{2}+\lambda_{t-1}^{2} D_{t}\left(s^{t}\right) \lambda_{t-1}^{2} \lambda_{t}^{2} w_{t-1}^{2}\right) \\
& w_{t}^{2}=\frac{1}{\left(1-\lambda_{t}^{2}\right) \lambda_{t-1}^{2} w_{t-1}^{2}} \frac{w_{t-1}^{2}}{\lambda_{t-1} w_{t-1}} \times \\
& \left(R\left(1-\lambda_{t-1}^{2}\right) \lambda_{t-1} w_{t-1} \lambda_{t-1}^{2} w_{t-1}^{2}+D_{t}\left(s^{t}\right) \lambda_{t-1}^{2} \lambda_{t-1}^{2} w_{t-1}^{2}+\lambda_{t-1}^{2} D_{t}\left(s^{t}\right) w_{t-1}^{1}\right) \\
& w_{t}^{2}=\frac{1}{\left(1-\lambda_{t}^{2}\right) \lambda_{t-1}^{2} w_{t-1}^{2}} \frac{w_{t-1}^{2}}{\lambda_{t-1} w_{t-1}} \times \\
& \left(R\left(1-\lambda_{t-1}^{2}\right) \lambda_{t-1} w_{t-1} \lambda_{t-1}^{2} w_{t-1}^{2}+D_{t}\left(s^{t}\right) \lambda_{t-1}^{2} \lambda_{t-1} w_{t-1}\right)
\end{aligned}
$$




$$
w_{t}^{2}=\frac{1}{\left(1-\lambda_{t}^{2}\right) \lambda_{t-1}^{2} w_{t-1}^{2}} w_{t-1}^{2}\left(R\left(1-\lambda_{t-1}^{2}\right) \lambda_{t-1}^{2} w_{t-1}^{2}+D_{t}\left(s^{t}\right) \lambda_{t-1}^{2}\right)
$$

Finally, this is equivalent to

$$
w_{t}^{2}=\frac{D_{t}\left(s^{t}\right)+R\left(1-\lambda_{t-1}^{2}\right) w_{t-1}^{2}}{\left(1-\lambda_{t}^{2}\right) w_{t-1}^{2}} w_{t-1}^{2}
$$

